Cinémas

Revue d'études cinématographiques

Journal of Film Studies

\title{
La double réception du film Métropolis de Fritz Lang
}

\section{Yves Laberge}

Volume 8, numéro 3, printemps 1998

Cinélekta 2

URI : https://id.erudit.org/iderudit/024757ar

DOI : https://doi.org/10.7202/024757ar

Aller au sommaire du numéro

Éditeur(s)

Cinémas

ISSN

1181-6945 (imprimé)

1705-6500 (numérique)

Découvrir la revue

Citer cet article

Laberge, Y. (1998). La double réception du film Métropolis de Fritz Lang. Cinémas, 8(3), 31-52. https://doi.org/10.7202/024757ar
Résumé de l'article

Le film Métropolis de Fritz Lang occupe une place exceptionnelle dans l'histoire du cinéma. Sorti une première fois en Allemagne en 1927, le film est relancé commercialement en 1984 après plus d'un demi-siècle. Métropolis est alors largement diffusé aux Etats-Unis et de par le monde dans une version adaptée au " goût du jour " (couleurs, trame sonore avec musique rock, promotion à vaste échelle, etc.). Après avoir défini les déterminations menant au succès ou à l'échec dans l'industrie du cinéma, l'auteur décrit les deux types de réception dont a fait l'objet ce " classique du cinéma » dans sa double « carrière ". 


\title{
La double réception du film Métropolis de Fritz Lang
}

\section{Yves Laberge}

\begin{abstract}
RÉSUMÉ
Le film Métropolis de Fritz Lang occupe une place exceptionnelle dans l'histoire du cinéma. Sorti une première fois en Allemagne en 1927, le film est relancé commercialement en 1984 après plus d'un demi-siècle. Métropolis est alors largement diffusé aux États-Unis et de par le monde dans une version adaptée au "goût du jour" (couleurs, trame sonore avec musique rock, promotion à vaste échelle, etc.). Après avoir défini les déterminations menant au succès ou à l'échec dans l'industrie du cinéma, l'auteur décrit les deux types de réception dont a fait l'objet ce "classique du cinéma" dans sa double "carrière".
\end{abstract}

\section{ABSTRACT}

Fritz Lang's Metropolis is considered as a masterpiece by many film historians. This science fiction production was presented first in 1927, during the silent era, and then in 1984, in a restored version with colors, rock music soundtrack, and a dynamic promotion campaign. The author considers what is success and what is fail in the film industry; afterwards, he sees if Metropolis succeeded or failed in its two carreers.

\section{Du succès dans les arts en général}

Pour le sociologue de l'art, la question de l'échec renvoie immédiatement à celle du succès. Ces étiquettes, on le sait, deviennent extrêrmement déterminantes dans la construction de ce que Howard Becker désigne comme la "réputation" d'un artiste au sein d'un "monde de l'art». Selon cet important 
sociologue américain, la sociologie de l'art doit étudier principalement l'organisation sociale, c'est-à-dire les liens entre les différents acteurs (créateurs, collaborateurs, distributeurs, critiques) participant à l'élaboration d'une ouvre d'art, sans s'attarder sur un artiste particulier ou sur la valeur esthétique des œuvres. La sociologie de Becker ne s'intéresse donc pas particulièrement à l'artiste, mais plus globalement aux réseaux (producteurs, fournisseurs, commanditaires, publicitaires, diffuseurs) dont l'artiste et son œuvre font intrinsèquement partie: "Nous pouvons examiner n'importe quel événement (lié à la production d'une œuvre d'art) et essayer de cerner le réseau, grand ou petit, dont l'action collective a permis à l'événement de se produire sous cette forme» (p. 364). Selon Becker, ce réseau de personnes qu'il désigne simplement comme étant un "monde de l'art» contribue à donner aux ouvres leur forme définitive. Il nous oblige dès lors à considérer chaque œuvre individuelle comme un véritable produit social: "Tous ces choix, opérés par un si grand nombre de participants, pendant la durée d'existence de l'œuvre, permettent d'affirmer que ce sont les mondes de l'art plutôt que les artistes qui font les œuvres" (p. 212). En résumé, Becker considère que "[...] l'objet de notre analyse n'est pas l'œuvre d'art en tant que réalité isolée (objet ou manifestation), mais l'ensemble des étapes de sa création et de sa recréation à mesure que des gens la découvrent et l'apprécient» (p. 226). Cette approche globale du phénomène de la création artistique donne à l'analyse qui en découle toute sa dimension sociologique.

À première vue, nous supposons que le succès ou l'échec permettent généralement de déterminer la qualité d'une œuvre artistique. Autrement dit, nous admettons que la qualité artistique d'un chef-d'œuvre peut dans certains cas passer inaperçue; mais nous supposons aussi que la plupart des ouvres célèbres et marquantes doivent d'abord leur réputation à leurs qualités intrinsèques. La qualité de l'œuvre serait donc, sinon garante de succès, au moins la preuvre d'un certain talent chez l'artiste qui cherche ultimement la réussite. Or, la notion de qualité représente en soi un problème si délicat pour de nombreux sociologues que ceux-ci préferent généralement l'éviter (Moulin, 1993). Dans son livre intitulé précisément Les Mondes de l'art, Howard 
Becker rappelle que "[...] la plupart des théories esthétiques et les courants traditionnels de la sociologie de l'art insistent sur la possibilité, et la nécessité, de juger la qualité des œuvres d'art " (p. 360). Mais ce jugement et cette sanction esthétiques des œuvres doivent ćtre laissés au critique ou à l'historien de l'art cette approche a été critiquée par le sociologue français Raymond Boudon.

Erving Goffman, un autre sociologue américain, adopte la même attitude face aux œuvres qu'il considère d'abord, au-delà de la qualité, du genre et de l'époque, comme des matériaux pour comprendre le social:

En fait, je n'établis aucune hiérarchie de valeur entre un bon et un mauvais roman, entre une pièce contemporaine et un drame antique, entre une bande dessinée et un opéra. Tous sont également utiles pour mettre au jour les caractéristiques d'un segment d'expérience. Si je me sers aussi bien des œuvres classiques et célèbres que des ceuvres mineures d'aujourd'hui, ce n'est pas parce que je pense qu'elles ont, chacune dans leur genre propre, une quelconque valeur culturelle. Les critiques et les commentateurs se réferent aux classiques d'un genre pour déceler l'importance de telle ou telle œuvre et sa nature d'œuvre d'art. Je me sers maladroitement des mêmes matériaux tout simplement parce qu'on a là quelque chose de facilement disponible, une sorte de fonds commun de l'expérience familière que l'auteur partage avec ses lecteurs (p. 24).

Au risque de donner l'impression de contourner à notre tour cette question épineuse de la qualité, nous nous contenterons dans un premier temps d'interroger la construction et la signification du succès et de l'échec des œuvres. Plutôt que de juger le succès artistique (ou comment l'atteindre), nous nous concentrerons sur la description de ses formes possibles en tentant d'en distinguer les différentes catégories. On peut identifier plusieurs sortes de succès et d'échec, car les œuvres ne font pas toujours l'unanimité auprès de leur public immédiat. Cette typologie du succès nous permettra ensuite de diagnostiquer la part de succès et d'échec qui a été réservée au film Métropolis au cours de ses deux carrières, soit en 1927 et en 1984. 


\section{Typologie du succès au cinéma}

Afın de mieux comprendre les différentes formes de succès possibles, nous préconisons dans ce texte des situations qui peuvent s'appliquer aux œuvres d'art en général, même si nous avons en tête, en les décrivant, des exemples tirés de l'histoire du cinéma. Sans être limitative, cette typologie peut contribuer à illustrer les formes possibles du succès que nous pouvons classer en sept catégories non exclusives: le succès symbolique, critique, populaire, d'estime, financier, à l'étranger, ou posthume. Évidemment, ces catégories se veulent purement pratiques et non exhaustives. De plus, une œuvre (un film) peut connaître, simultanément ou successivement, une ou plusieurs formes de succès. Décrivons-les brièvement.

\section{- LE SUCCESS SYMBOLIQUE}

Le succès symbolique survient lorsqu'une ouvre est primée par un jury à l'occasion d'un concours, d'un festival, d'une rétrospective, ou à la suite de toute autre épreuve éliminatoire. Lobtention d'une subvention peut également constituer en soi une forme tangible de récompense symbolique. On pourrait bien sûr ajouter plusieurs niveaux ou sous-catégories, dans la mesure où certains prix valent plus que d'autres: certains festivals sont davantage publicisés ou reconnus depuis plus longtemps, tout comme certains concours semblent plus élitistes que d'autres. Mais, comme on s'en doute, il advient aussi que des œuvres primées semblent curieusement dénuées de valeur esthétique; elles ennuient les critiques et déroutent une partie du grand public qui, dit-on, croirait aveuglément au jugement des critiques et à la sanction des récompenses symboliques. Ces mêmes ouvres ne remplissent pas les salles et ne font pas leurs frais, elles ne connaissent aucune carrière internationale et peuvent être oubliées à jamais. Les jurys, comme les artistes, peuvent s'égarer, se méprendre. Le public (dont on entend parfois dire qu'il ne se trompe jamais!) peut sortir déçu d'une manifestation artistique particulièrement vantée et de laquelle il attendait beaucoup. Par contre, comme les jurys se composent souvent de membres du milieu artistique, les récipiendaires peuvent toujours clamer qu'ils ont été jugés par leurs pairs et 
non par un public insensible, accordant ainsi plus d'importance à leur récompense symbolique qu'à leur échec critique ou financier. On retiendra enfin que le succès symbolique survient parfois au moment où l'œuvre demeure encore inédite et non rentabilisée (par exemple lors de présentations dans la sélection du festival de Cannes, qui exige que les œuvres en compétition soient inédites et projetées en première mondiale). Dans ces cas, le caractère secret da la sanction empêche pour un bref moment tout jugement qui serait contradictoire et peut ainsi donner au succès symbolique beaucoup plus de poids.

\section{— LE SUCCÈS CRITIQUE}

Si le succès symbolique contribue à lancer une œuvre et doter celle-ci d'une certaine dose de célébrité, le succès critique peut également jouer un rôle tout aussi déterminant. Les critiques locale, nationale, étrangère, internationale peuvent, chacune à sa façon, donner un poids, une portée à l'œuvre. Qu'elle soit spécialisée ou qu'elle reste dans le domaine du vague (en reproduisant par exemple le texte d'un dossier de presse ou alors des extraits d'autres critiques à défaut de proposer une véritable analyse de fond), la critique servira peut-être d'incitatif, de catalyseur, afin de convaincre un certain public de consommer l'œuvre ou de lui accorder son attention (acheter une reproduction d'une affiche, assister à une projection d'un film, prendre connaissance d'autres critiques). Le verdict d'un critique influent et reconnu contribue dans tous les cas à faire connaître l'œuvre en question. En soi, la critique ne doit pas nécessairement être bonne ou excellente pour motiver des réactions auprès du public; les critiques destructrices et les scandales, dans la presse et dans certains milieux, font vendre tout aussi bien. Le succès critique devient déterminant dans la mesure où cette faveur permet à l'œuvre de recevoir une publicité gratuite parfois largement diffusée qui peut mener à une consommation directe. Les producteurs compensent souvent l'absence de succès critique par une intensification de la promotion et de la publicité. 


\section{- LE SUCCÈS POPULAIRE}

Le succès populaire constitue une sorte de norme, un but apparemment honorable et légitime qui réjouit tous les producteurs, artisans et participants d'une ouvre moyenne. Dans une sorte d'heureux enchaînement, les critiques accueillent assez favorablement l'œuvre, le public en apprécie les qualités, la publicité semble produire les effets escomptés, et le film (ou l'œuvre d'art) semble pouvoir rencontrer ses frais, sans nécessairement générer des profits considérables. Il s'agit somme toute d'un cas moyen, bon sans être particulièrement remarquable, imparfait bien sûr, mais sans être banal. Peu importe les raisons, l'œuvre "fonctionne bien". Le succès populaire résulte d'une sorte de mélange à dose moyenne de plusieurs types de succès (critique, financier et / ou d'estime).

\section{- LE SUCCÈS D'ESTIME}

Le succès d'estime se situe entre le succès populaire et le succès symbolique ou critique; il participe de ces deux dernières catégories. Il ne s'agit pas ici d'œuvres financièrement "rentables", au sens matériel du terme bien qu'elles contribuent à faire connaître l'artiste, à lui attirer respect, considération et éventuellement d'autres projets ou collaborations. Dans ce type de réception, les spécialistes (jurys, critiques, autres artistes et "connaisseurs ») ont apprécié une ouvre que le grand public a boudée ou n'a pas comprise. Évidemment, certains artistes peuvent toujours, avec parfois une certaine dose de prétention ou d'opportunisme, se flatter de leurs nombreux succès d'estime et se consoler ainsi de leurs échecs financiers ou de l'incompréhension du grand public, ce qui, en outre, leur assurera peutêtre l'étiquette d' "artiste incompris ».

\section{- LE SUCCÈS FINANCIER}

Le succès financier se mesure facilement en termes de profits. Cette évaluation doit cependant tenir compte des coûts de production et de postproduction, des frais engagés dans les campagnes publicitaires et promotionnelles. Une superproduction au coût de 20 millions $\$$ qui amasse des recettes de 18 millions $\$$ sera déficitaire à court terme malgré l'apparence de fortes ren- 
trées d'argent et un succès populaire considérable. Les productions plus modestes, les films d'auteur souvent réduits à de petits moyens de production et destinés aux salles d'art et d'essai, ont quelquefois plus de chances de rencontrer leurs frais à moyen terme. Dans plusieurs cas, les meilleures recettes au cinéma proviennent, on le comprendra facilement, d'un film à petit budget qui connaît un grand succès (ou un long succès) et génère de larges bénéfices.

\section{- LE SUCCĖS À L'ÉTRANGER \\ ET LE SUCCÈS INTERNATIONAL}

Le succès à l'étranger apparaît parfois comme un phénomène étrange: pourquoi tel film allemand remporte-t-il un tel succès au Japon '? Souvent, le succès à l'étranger signifiera une sorte de consécration autant pour les créateurs que pour la critique. Lorsque ces vagues de succès se répandent, d'un pays à l'autre, et ne constituent plus un cas isolé, puisque l'on dépasse le réseau de ce que l'on pourrait désigner comme le "marché naturel extérieur" (c'est-à-dire la voie que suivent habituellement toutes les ouvres d'un circuit artistique), on peut dès lors parler de succès international. Par ailleurs, certaines ceuvres connaissent un succès qui semble, jusqu'à un certain point, éternel: le film Mort à Venise du cinéaste italien Luchino Visconti est présenté pratiquement sans interruption dans différentes salles d'art et d'essai de Paris depuis sa sortie en 1971.

\section{- LE SUCCÈS POSTHUME OU TARDIF}

Enfin, lorsque tous ces publics semblent avoir oublié (ou ignoré) une œuvre redécouverte par la suite, on peut parler alors de succès tardif, parfois posthume (longtemps après la carrière normale de l'œuvre ou après le décès de l'artiste). C'est ainsi que l'on découvre des œuvres originales et dignes d'intérêt d'un artiste resté longtemps méconnu et qui connaît tardivement la célébrité. Les premiers films du cinéaste allemand Rainer Werner Fassbinder ont subi ce sort: ses 30 premiers longs métrages ont été à peu près ignorés hors d'Allemagne avant de bénéficier de la popularité internationale des 10 derniers films créés par le réalisateur. Soulignons aussi le cas du film La Règle du jeu 
(1939) de Jean Renoir, maintes fois lancé et relancé sans succès, et qui connaît finalement un accueil enthousiaste du public un quart de siècle après sa sortie initiale ${ }^{2}$.

\section{- CONCLUSION PROVISOIRE}

Cette typologie nous permet surtout de constater qu'en définitive, le succès comme l'échec ne sont jamais complets puisqu'il existe plusieurs formes alternatives de réussite pour une œuvre durant toute l'histoire de sa réception. Cependant, la façon dont l’euvre sera accueillie demeure en soi déterminante (connaître un succès financier n'équivaut pas à un succès d'estime, même si un certain type de succès peut ensuite mener à un autre). Ainsi, certaines superproductions ultra-commerciales remportent un grand succès financier, tout en étant controversées chez une partie de la critique (ce qui n'empêche pas l'acteur Sylvester Stalone et le réalisateur américain Oliver Stone d'obtenir une reconnaissance symbolique de la part de l'État français). Il importe toutefois d'établir clairement, dans la mesure du possible, la forme et l'impact d'un succès ou d'un échec pour l'artiste et pour son cuvre.

L'échec ne se réduit pas forcément à l'absence totale ou partielle de toute forme de succès. L'échec survient lorsque l'œuvre ne semble pas à la hauteur des espoirs que ses producteurs lui ont conférés. Pour cette raison, les trop grandes attentes risquent souvent de laisser à leurs producteurs comme au public une impression d'échec à propos d'une œuvre pourtant reçue honorablement.

\section{La légitimation de la qualité des œuvres}

Les jugements sur l'importance et la qualité des ouvres posent toujours le même problème: comment déterminer les «bons» films, les cuvres importantes ou significatives? On pourrait procéder de différentes façons: entreprendre des sondages à la sortie des salles pour demander aux spectateurs ce qu'ils pensent du film qu'ils viennent de visionner; se fier aux commentaires dans les journaux ou aux critiques dans les revues plus spécialisées; vérifier si le film a été rentable pour ses producteurs, s'il a tenu longtemps l'affiche ou s'il a été retiré au bout d'une semaine. Tous ces sondages tentent, imparfaitement, de 
mesurer l'ampleur du succès d'une œuvre. L'analyse économique globale permet, par exemple, grâce à la comparaison d'indicateurs comptables (nombre d'entrées, niveau des recettes), de juger du succès d'un film. Il existe cependant une foule d'autres facteurs (la publicité, les autres films à l'affiche, la période de l'année) pouvant influencer et relativiser ces observations. La plupart des salles commerciales présentent des nouveautés qui restent habituellement à l'affiche aussi longtemps qu'elles rapportent (ou jusqu'à ce qu'un titre plus prometteur ne devienne disponible); elles programment rarement des œuvres, même excellentes, datant de plus de deux ans. Or, les pratiques commerciales liées aux impératifs de rentabilité ne devraient pas nous empêcher de repérer de nombreux chefs-d'œuvre transcendant les barrières du temps pour devenir universels et virtuellement éternels. Hélas, bon nombre de "classiques» inaccessibles du cinéma demeurent inconnus des nouvelles générations. Ce fut pendant longtemps le cas de Métropolis avant sa seconde sortie commerciale.

\section{Une deuxième carrière après un demi-siècle}

Ces observations sur la courte vie d'un film dans le circuit des salles commerciales permettent de saisir le caractère exceptionnel de la seconde sortie d'un film datant de l'époque du muet: Métropolis de Fritz Lang. Tourné en Allemagne en 1925 et projeté pour la première fois en janvier 1927, le film est relancé pour une deuxième fois dans le circuit des salles commerciales à l'été 1984. Cette seconde sortie pose une question intéressante et inhabituelle à la sociologie du cinéma dans la mesure où elle permet de vérifier la résistance et la fragilité d'un contexte de production d'origine devant une appropriation interprétative qui lui est étrangère. Ce film, lié au contexte de sa production (l'Allemagne de l'entre-deux-guerres), ressort commercialement (et non dans le circuit restreint des cinémathèques et des salles d'art et d'essai) un demi-siècle plus tard devant un nouveau public. Il est accompagné d'une campagne de promotion comparable à celle des nouveautés hollywoodiennes: affiche, disque et cassette de la bande musicale, vidéoclip de la chanson Here's my Heart (interprétée par la chanteuse Bonnie Tyler et illustrée 


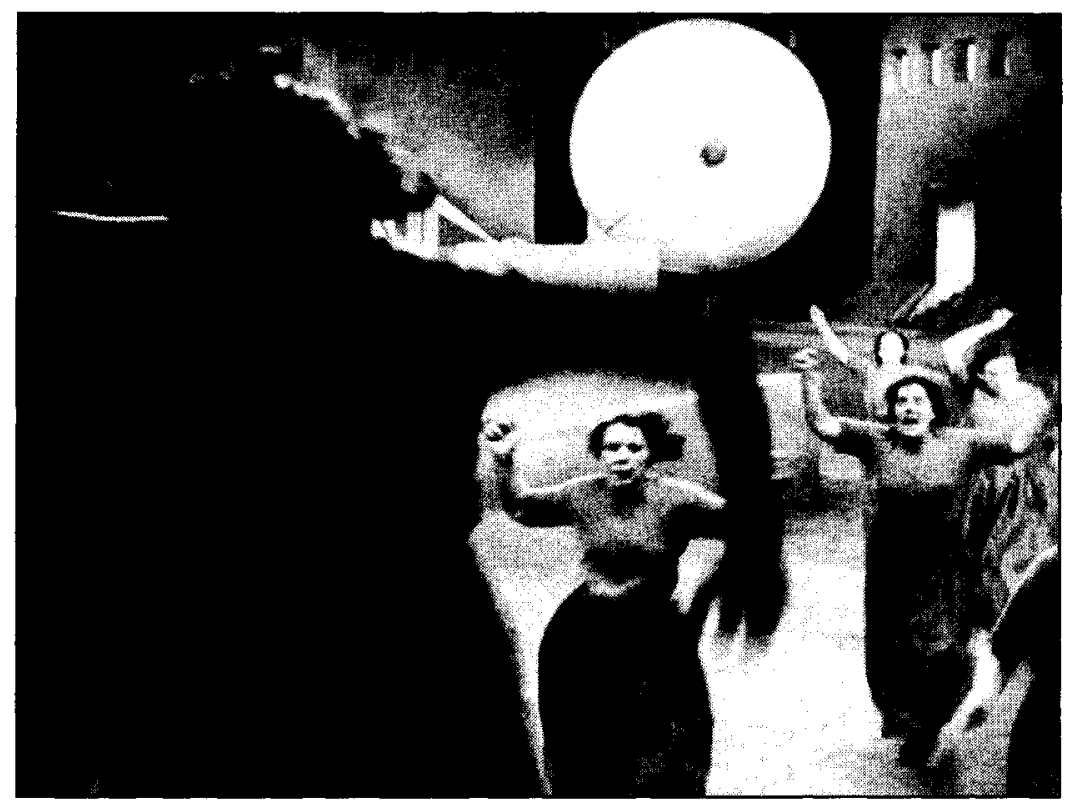

Métropolis de Fritz Lang (1927)

Collection Cinémathèque québécoise

par les images du film Métropolis), entrevues avec l'instigateur du projet, critiques dans les journaux et revues spécialisées, etc. À quelle réception cette seconde sortie livre-t-elle le film? A quelle motivation correspond cette deuxième vie commerciale de Métropolis? Avant de répondre à ces questions, examinons d'abord les circonstances ayant entouré la production initiale et la première sortie de ce film.

\section{Situation historique de Métropolis}

Comme il arrive souvent à la sortie de nombreuses superproductions de l'histoire du cinéma - dans ce cas-ci "[...] la plus coûteuse production du cinéma allemand muet " (Courtade, p. 154 et Marie, p. 6), c'est-à-dire plus d'un million de marks de l'époque (Passek, 1978, p. 82) -, les commentaires et les critiques de l'époque sont généralement hostiles envers Métropolis. On ne trouve que des opinions franchement improbatrices dans les huit extraits de commentaires plus ou moins anciens qui ont été rassemblés dans une notice sur Métropolis et figurant parmi 
une centaine d'autres critiques de films réunies dans un important catalogue consacré au cinéma allemand de cette période (Passek, 1978, p. 82-85). Par ailleurs, le film n'a pas fait ses frais, générant un déficit de plusieurs millions et causant presque la faillite de sa maison de production allemande, la UFA (Eibel, p. 36 et 342-343). De plus, comme si ces problèmes ne suffisaient pas, un procès pour plagiat, resté sans suite, a été intenté en 1927 contre la romancière Thea Von Harbou, coscénariste de Métropolis et épouse de Fritz Lang. Mais avant d'examiner plus en détails quelques critiques négatives du film, il convient de montrer à quel point son auteur a su gagner progressivement le respect des historiens du cinéma et obtenir aujourd'hui une reconnaissance pratiquement universelle.

Fritz Lang demeure encore de nos jours un cinéaste admiré. Dans un article de deux colonnes signé par Gérard Legrand, qui accorde d'ailleurs plus d'importance à la période états-unienne (1935-1956) qu’à la période allemande (1918-1933) de Lang, le récent Dictionnaire du cinéma (Passek, 1991) le présente encore maintenant comme un "cinéaste américain d'origine autrichienne", ce qui sans être faux occulte à première vue sa contribution essentielle à l'histoire du cinéma allemand, contribution pourtant louangée dans ce même commentaire (p. 390). D'ailleurs, "[...] sa période allemande a toujours rencontré une certaine unanimité[...]», selon Michel Marie, qui affirme lui aussi que " [..] Fritz Lang est aujourd'hui encore considéré comme un maître du septième art [...]" (p. 7). Sa longue carrière serait entièrement "[...] jalonnée de chefs-d'œuvre qui sont en même temps devenus ceux de l'histoire du cinéma [...]", lit-on par ailleurs dans un article fort élogieux occupant deux pages de $L ' E n-$ cyclopédie Universalis (Brion, p. 432).

\section{L'intrigue de Métropolis}

Avant de poursuivre, rappelons brièvement quelques éléments du scénario de Métropolis. Nous trouvons, dans le monumental Guide des films dirigé par l'historien Jean Tulard, une synthèse concise et claire du film:

Métropolis, la ville de demain où s'oppose à une caste de privilégiés, une masse d'esclaves parqués dans des 
souterrains et soumis à des cadences infernales. La révolte gronde, mais elle est apaisée par la jeune Maria. Pour contrer l'influence de cette dernière, le chef de la cité demande à Rotwang de lui construire un robot à l'image de Maria, afin de pousser les ouvriers à la révolte. Mais son fils aime la vraie Maria. Ils s'opposeront à l'andrö̈de qui déclenche une véritable catastrophe. Tout s'achèvera par la réconciliation du travail et du capital (p. 178).

Lhistorien Jean Tulard, qui a rédigé lui-même cette notice, présente Métropolis comme "un sommet du cinéma allemand" et lui accorde la cote maximale de quatre étoiles réservée dans son livre aux "classiques" de l'histoire du cinéma.

Malgré la réputation du film Métropolis, peu de gens le désignent cependant comme "le plus grand film" de Lang, même s'il figure certainement parmi ses plus célèbres. Peu de cinéphiles n'ont jamais entendu parler de Métropolis. Par contre, on s'accorde généralement pour considérer $M$ le maudit (1931) comme étant le meilleur long métrage de Fritz Lang. Ce dernier est d'ailleurs celui sur lequel les critiques ont le plus écrit (Laberge, 1994). Lang lui-même préférait d'entre tous ses films son premier long métrage parlant ( $M$ le maudit) et il était en revanche très sévère vis-à-vis Métropolis. Il déclarait à ce propos dans une entrevue publiée en 1959 dans la revue Cahiers du cinéma: "Personnellement, je n'aime pas beaucoup le film. On ne peut plus dire aujourd'hui que le cœur est le médiateur entre la main et le cerveau. C'est faux, la conclusion est fausse, je ne l'acceptais déjà pas quand je réalisais le film " (cité dans Passek, 1978, p. 82).

\section{La réception critique de Métropolis à sa première sortie}

On retient des quelques critiques d'époque des jugements généralement sévères qui jettent des doutes sur la part de succès de la première version de Métropolis. Ainsi, un critique allemand du journal spécialisé Filmschau a écrit ce commentaire condamnatoire au moment de sa première sortie:

Fritz Lang est considéré comme le plus doué des metteurs en scène allemands. Cependant, on est tenté de 
dire le contraire après avoir vu Métropolis, ce film annoncé à grand fracas comme étant le plus grand chefd'œuvre de tous les temps, un film que tout le monde attendait avec impatience. Bien sûr, la mise en scène est digne des autres films de Fritz Lang. Mais le choix du sujet passe avant la mise en scène, et de ce point de vue là, Métropolis est un échec. Évidemment, c'est le plus cher des films allemands, c'est le plus grand des films allemands, etc., la presse ne trouve d'ailleurs pas assez de superlatifs pour le définir: 50000 figurants (exactement 48733, d'après le programme) et de fait, sur le plan technique, c'est indéniablement un chef-d'œuvre. Mais cela n'empêche pas que Métropolis soit un navet, un très noble navet, mais un navet tout de même ${ }^{3}$.

Un autre critique de la même période, Paul Ickes, écrit dans le même journal :

Je ne peux m'empêcher de clamer que c'est de la bêtise, du verbiage, du suspense... et rien d'autre! [...] Le roman et le scénario manquent totalement d'imagination, alors que c'était justement l'élément le plus important pour un tel film. [...] C'est la chose la plus décevante et la plus discutable que j'aie vue depuis longtemps.

Un autre journaliste allemand reproche à l'intrigue de ne pas correspondre à la vision idéalisée que l'on se faisait alors des machines du futur: " [...] la technique de l'avenir ne devrait pas faire des hommes des esclaves, mais les maîtres des machines. Ces machines, de plus, seraient automatiques: un seul ouvrier intelligent suffirait à assurer le fonctionnement de toutes les manettes à la fois" (cité dans Eisner, p. 103).

En France, on peut lire dans l'éphémère revue cinématographique Les Annales (à ne pas confondre avec la célèbre revue d'histoire, d'économie et de société) du 25 novembre 1927 un commentaire tout aussi mordant que les précédents : "D'un intérêt artistique nul, ce scénario [est] d'une sottise absolue [... ${ }^{4}$.»

Le grand romancier anglais $\mathrm{H}$. G. Wells fut lui aussi très sévère face au filrn d'anticipation de Lang:

Je viens de voir un film stupide. Je ne crois pas qu'il y ait moyen d'en faire un qui soit plus bête que celui-là. 
Il s'appelle Métropolis et est une production de l'UFA, une grande société cinématographique allemande, qui vante à son public les sommes qu'elle a dépensées pour le réaliser. Et malheureusement le film est une synthèse de toutes les bêtises et toutes les platitudes que nous connaissions, agrémentées d'une sauce sentimentale unique en son genre. Le pis est que ce film idiot, sentimental et manquant totalement d'imagination, gaspille de très belles possibilités. Je pensais que les Allemands étaient entreprenants, j'en doute maintenant. Je suis terriblement déçu (cité dans Passek, 1978, p. 82).

On rencontre en fait peu de commentaires favorables à Métropolis à cette époque, sauf peut-être des opinions mitigées de deux créateurs, soit celles du romancier Jules Romains et du jeune Luis Buñuel (alors encore inconnu). Romains écrit: «La technique de Métropolis n'est pas impeccable, ni égale. Le style pêche trop souvent par redondance ou amplification [...]. Mais n'importe quel spectateur en sent la force, la nouveauté, l'autorité " (cité dans Passek, 1978, p. 84). La critique de Buñuel reproche au film son sentimentalisme, mais en reconnaît également l'impressionnante mise en scène: "[...] Métropolis comblera tous les vœux, nous émerveillera comme le plus merveilleux livre d'images qui se puisse composer... quelle enthousiasmante symphonie du mouvement!" (cité dans Eisner, p. 106).

La première sortie de Métropolis connaît donc de graves difficultés financières en raison des coûts élevés de production (deux années de travail et plusieurs milliers de personnes employées) et des (relativement) faibles profits à sa sortie. De plus, on note de nombreuses critiques défavorables. Un échec financier se double donc d'un échec critique auquel s'oppose un faible succès d'estime. Pourtant, ce film réalisé par un cinéaste important et mal reçu à sa première sortie s'installe dans l'histoire du cinéma comme un grand film d'anticipation. Sans doute, les films antérieurs et subséquents de Fritz Lang ont-ils servi à établir et la notoriété du réalisateur et la réputation de son œuvre. En conclusion, comme le film Métropolis est néanmoins passé à l'histoire du cinéma, on peut donc affirmer qu'il a connu un succès tardif ou posthume. Toutefois, les circonstances entourant ce succès posthume demandent à être examinées. 


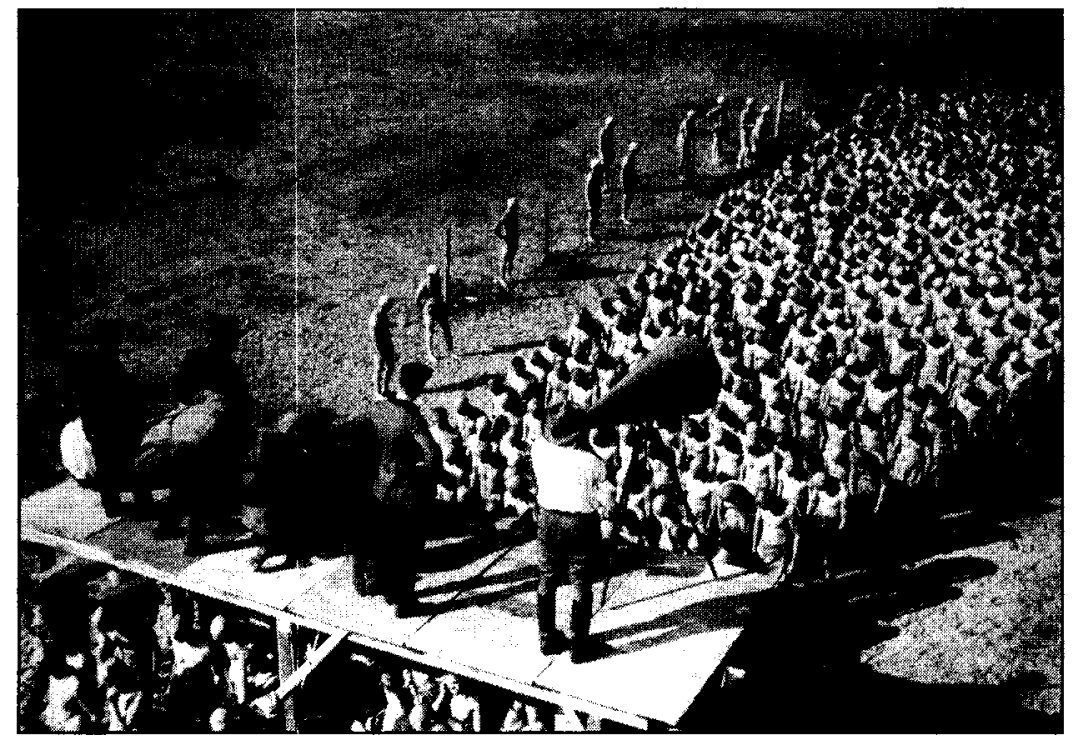

\section{Métropolis de Fritz Lang (1927)}

Collection Cinémathèque québécoise

\section{La seconde sortie de Métropolis}

De nos jours, plus de gens ont eu l'occasion de voir la nouvelle version sonorisée et colorée de Métropolis que l'ancienne, muette et en noir et blanc, datant de 1927. Cette entreprise de re-découverte, lancée aux États-Unis, a été menée par le producteur américain Giorgio Moroder et financée avec des capitaux américains. Cette version "Moroder » est identifiée comme étant une coproduction germano-américaine et non plus seulement allemande comme la version de 1927.

L'étude de cette seconde sortie, sous l'angle de sa réception critique, peut donner lieu à d'intéressantes réflexions. La question des publics et des réseaux d'une œuvre donnée mérite en soi une attention spéciale; nous n'évoquerons cependant que brièvement cet aspect. On peut aussi se demander ce qui s'est passé durant le demi-siècle qui a séparé les deux sorties de ce film afin de comprendre à quoi correspondent, historiquement et socialement, chacune de ces deux sorties. Du point de vue de l'histoire technique du cinéma, la fin des années vingt marque au cinéma la fin du muet et l'avènement du parlant. Après 
1930, la majorité des films de long métrage destinés aux écrans commerciaux sont désormais parlants.

Entre-temps, malgré les critiques sévères à l'endroit du film Métropolis dont la carrière est écourtée, entre autres, par l'arrivée progressive des films parlants et l'engouement du public pour cette nouvelle formule, on peut néanmoins constater que Fritz Lang lui-même demeure un cinéaste respecté chez qui on reconnaît le génie. Lang fut pourtant souvent critiqué en Allemagne, surtout autour de 1930 , mais on lui reprochait plutôt son audace et les excès de sa personnalité. Si on apprécie peu sa personne ou le sujet de ses films, on lui reconnaît un grand savoirfaire et un professionnalisme inattaquable. Si Métropolis n'est pas un film parfait, son créateur reste un véritable auteur et un grand cinéaste.

\section{La réception de Métropolis en 1984}

La nouvelle version de Métropolis est présentée en première mondiale à la Quinzaine des réalisateurs du festival de Cannes, en mai 1984. Si la revue française les Cahiers du cinéma passe sous silence cet événement, la revue Positify consacre un long et excellent article signé Brigitte Cieutat. L'auteure y évoque tout d'abord «[...] les moyens publicitaires mis en ouvre et les nombreux articles que l'ensemble de la presse a consacrés [...]" au film durant l'été 1984, avant d'exprimer une opinion mitigée. Cette reconstitution,
[...] retrouvant des scènes ou des séquences inconnues du public, est l'aboutissement d'une recherche fort res- pectable, mais triturer une cuvre de la sorte, sous pré- texte que Lang n'en avait pas laissé de montage défini- tif, l'adapter à des rythmes qui lui sont totalement étrangers pour la rendre plus accessible aux jeunes, relève d'une manipulation difficilement admissible (Cieutat, p. 11).

Critique au journal Le Monde, Hervé Guibert pose la même alternative dès le titre de son article: "Fritz Lang défiguré ou magnifié?». Le journaliste, plutôt favorable à l'entreprise de "restauration" de Moroder, affirme que l'œuvre de Lang gagne à ce traitement: "Non seulement son film résiste totalement, 
mais cette version Moroder le magnifie et donne envie de retourner sur-le-champ à l'original. Le lifting, en effet, ne devrait pas tenir longtemps " (Guibert, p. 11).

Par ailleurs, le mensuel Le Monde diplomatique du mois d'août 1984 consacre au film une pleine page avec trois articles. Le premier comprend des extraits consacrés au film Métropolis et à sa signification historique, tirés du livre (fort controversé) $D e$ Caligari à Hitler (1947) de Siegfried Kracauer. Un deuxième article, assez approximatif, signé François Garçon et intitulé "L'annonce du règne hitlérien », est précédé d'un article de Ignacio Ramonet intitulé "Métropolis, l'amour des classes». Ces trois articles s'attardent sur les significations politiques possibles de cette seconde sortie de Métropolis, un film par ailleurs ambigu d'un point de vue idéologique.

Notre propre recherche a démontré deux aspects importants à propos de cette nouvelle version: premièrement, la version de Moroder omet de nombreux plans dans plusieurs séquences; deuxièmement, lorsqu'elles sont juxtaposées, ces omissions imperceptibles modifient le sens du film de Lang en légitimant les actions subversives du personnage du patron, et en modifiant les enjeux de la révolte des ouvriers (Laberge, 1984). Le problème du "nouveau sens" de la version "Moroder" de Métropolis pourrait en soi donner lieu à des recherches beaucoup plus poussées. Restons-en simplement à la question du succès de l'œuvre.

La nouvelle version de Métropolis a sûrement contribué à faire connaître Fritz Lang auprès de plusieurs générations de cinéphiles qui n'avaient probablement jamais entendu parler de lui. Ce fut aussi une occasion de revoir sur grand écran et dans des salles commerciales un classique du cinéma muet (dans une version musicale avec synthétiseurs et chansons rock en dolby stéréo!), ce qui n'est hélas pas très fréquent. Cette version se présentait dans une formule assez inhabituelle: ce film d'autrefois mais d'anticipation, doublé d'une trame sonore contemporaine, n'est déjà plus, après ces rénovations, l'œuvre datant de plus d'un demi-siècle. La critique récente a quelquefois reproché au film son manque d'authenticité (la couleur et la musique n'ont pas été choisies par Lang) et s'est parfois interrogée sur le sens que 
l'histoire a laissé peser sur l'œuvre allemande de ce cinéaste allemand qui a pourtant fui le troisième Reich dès 1933, mais dont les films auraient plu au Führer (Lang, cité par Kracauer, p. 181).

Quant au type de succès réservé à la seconde carrière de Métropolis, on remarque d'abord que les arguments des critiques de 1927 ne servent plus aujourd'hui : personne n'est dérangé ou choqué par les excès de sentimentalisme ou la prétendue inadéquation de la vision anticipatrice du futur proposée par les auteurs du film. Peu de critiques rappellent aujourd'hui les arguments des contempteurs des années 1920. Au lendemain de sa présentation à la Quinzaine des réalisateurs du festival de Cannes de 1984 (cette première sélection constitue en soi une épreuve symbolique réussie), le quotidien Le Matin de Paris du 14 mai 1984 titrait : "La Quinzaine des réalisateurs: Métropolis sauce rock». L'article du journaliste Alain Riou amorçait d'entrée de jeu le débat entre les partisans et les détracteurs de la nouvelle version de Moroder. Aucun débat ne touchera le contenu même du film, désormais reconnu unanimement comme un chef-d'œuvre indiscutable; la plupart des critiques porteront maintenant sur la légitimité du travail de reconstitution et de recréation de l'œuvre de Fritz Lang par le producteur G. Moroder. Le regard critique se porte sur le travail de restauration du film en situant les enjeux du débat.

Pourtant, comme nous l'avons rappelé plus haut et ailleurs (Laberge, 1985 et 1997), la version "Moroder", en plus de transformer esthétiquement une œuvre de 1927 en lui ajoutant couleurs et musique d'une autre époque, escamote plusieurs plans et répliques pourtant contenus dans les versions antérieures à 1984. Non seulement aucun critique ne s'est aperçu de ce problème, mais plus encore, depuis cette nouvelle sortie, la version "Moroder" du film Métropolis est désormais programmée dans les salles d'art et d'essai, dans de nombreuses cinémathèques et à la télévision '.

Des théoriciens importants du cinéma se sont également penchés sur le cas de la nouvelle version de Métropolis produite par G. Moroder sans remarquer quoi que ce soit à propos des nombreuses modifications et des retranchements apportés à la nou- 
velle version du film (Odin, 1988 et Metz, 1991). Ainsi, pour Christian Metz, "[...] c'est le même film [...]” (p. 100).

De plus, cette version "Moroder» a été rapidement disponible en cassette vidéo, autant pour l'achat que pour la location, alors que la version muette, qui existe également en format vidéo, a été longtemps difficile d'accès. Ainsi, la version sonorisée demeure plus accessible, plus populaire et plus vue. On en conclut que cette version modernisée, assurément non conforme au projet initial de Fritz Lang, a tout de même reçu, en plus d'un succès critique et d'un succès populaire, un indéniable succès à long terme. Cette nouvelle version est désormais devenue la référence incontournable chez le nouveau public du film Métropolis.

Il faudrait certainement s'interroger sur les causes de cette situation: comment et pourquoi la version incomplète du film Métropolis, présentée par son producteur comme étant la plus complète (Moroder, cité par Borie, p. 69), est-elle devenue une référence? Deux raisons pourraient expliquer ce phénomène: l'attrait de la nouveauté et la diffusion efficace. D'abord, il faut admettre que cette version sonorisée a été largement publicisée comme étant un classique du cinéma de science-fiction et d'anticipation. Cette nouvelle version a beaucoup circulé depuis plus de 10 ans. D'abord dans les festivals et les circuits commerciaux des salles d'exclusivités (au même titre que les plus récentes nouveautés de Hollywood), ensuite dans les circuits parallèles réservés aux publics plus avertis. Deuxièmement, un film aussi séduisant, capable d'intéresser un auditoire varié composé de critiques, de cinćphiles, de locateurs de cassettes vidéo, de téléspectateurs, en vient facilement à se substituer à un film muet portant le même titre et qui ne bénéficie plus que d'un lointain succès obtenu non pas à sa première sortie, mais au fil des décennies, à mesure que l'œuvre de Lang trouve sa place au sein de l'histoire du cinéma. Ironiquement, cette réputation de «classique du cinéma muet», que l'on accorde progressivement à Métropolis, sert désormais à promouvoir la nouvelle version "Moroder". Les spectateurs, intéressés au caractère subversif du film dans sa version muette, n'ont plus la chance de voir qu'une "restauration" qui contredit l'œuvre de Fritz Lang. Comme les structures de distribution cinématographique avantagent les 
productions (ou les coproductions) américaines (Guback, 1976), on comprendra dès lors comment la version "Moroder" de Métropolis a pu réussir à obtenir un succès aussi décisif. La controverse que certains critiques ont soulevée à propos de la version modernisée ne compense pas l'étonnante reconnaissance institutionnelle (salles, vidéo, cinémathèques) que celle-ci a néanmoins obtenue.

La question du succès et de l'échec permet de comparer l'accueil d'un film qui, exceptionnellement, connaît deux carrières séparées de plus d'un demi-siècle, dont une posthume à son auteur. Une typologie du succès nous donne des outils conceptuels simples et utiles pour examiner la réception de chaque version du film.

Le film Métropolis a été un échec critique et financier lors de sa sortie initiale en 1927. Néanmoins, le film et son réalisateur sont progressivement passés à l'histoire (du cinéma) au fur et à mesure que des travaux sont consacrés au cinéma expressionniste allemand et aux films subséquents de Fritz Lang. Par ailleurs, malgré une certaine controverse entourant la légitimité (et non la qualité) de l'entreprise de restauration du film Métropolis par le producteur américain G. Moroder, une nouvelle version sonorisée connaît un succès populaire; la critique, le public et le milieu du cinéma lui accordent un accueil honorable, plus important qu'un succès d'estime, mais sans nécessairement battre des records. Personne ne remarque les limites et les subtilisations de cette version "Moroder", qui passe à l'histoire en devenant désormais la version de référence dans de nombreux réseaux de diffusion des films (salles de reprises, télévision, cassette vidéo).

En laissant de côté l'analyse directe de l'œuvre (mais tout en y faisant référence à l'occasion), la sociologie du cinéma nous donne l'occasion d'étudier le processus de légitimation d'un film fondé sur le succès qui, se construisant dans le temps et grâce à une large diffusion à travers tout le réseau des salles de projection, contribue à assurer la consécration. C'est ce qui a sans doute été réussi, contre toute attente, par le producteur G. Moroder". 


\section{NOTES}

1 On se souviendra du cas inusité du film muet De l'aube à minuit (1920) relaté par Lotte Eisner dans le documentaire qui lui est consacré intitulé La mort n'a pas voulu de moi (1982). Le film De l'aube à minuit, adapté par Karl-Heinz Martin d'une pièce de Georg Kaiser, était considéré comme perdu depuis des années jusqu'à ce qu'une copie soit retrouvée au Japon où l'ouvre avait à l'époque remporté un grand succès.

2 Voir le livre dirigé par Y. Karsunke, Fassbinder (Paris: Rivages, 1986). Sur Renoir, voir notre étude sur La Règle du jeu dans Communication Information, vol. 16, n" 2 (1995), p. 235 et sq.

3 Commentaire anonyme paru dans Filmschau en 1927 et cité dans le collecrif Fantastique et réalisme dans le cinéma allemand 1912-1933 (Bruxelles: Cinémathèque Royale de Belgique, 1969, p. 44).

4 Extrait cité brièvement dans l'article de François Garçon, "L'annonce du règne hitlérien ", Le Monde diplomatique (août 1984), p. 24. On trouve dans le même article un court extrait de la critique du futur cinéaste Luis Buñuel, mais dans lequel on ne transcrit que les propos négatifs de sa pensée, alors qu'en fait, celui-ci a été impressionné par le film, malgré ses faiblesses de ton et son sentimentalisme. Voir Lotte $\mathrm{H}$. Eisner, Fritz Lang (Paris: Flammarion, 1988, p. 106). Plus tard, Buñuel admit que les premiers films muets de Lang avaient joué un rôle décisif dans l'émergence de sa vocation de réalisateur. Voir Luis Buñuel, Mon dernier soupir (Paris: Robert Laffont et Ramsay, 1986, p. 242)

5 C'est le cas au Québec à la Cinémathèque québécoise de Montréal dans sa programmation régulière, et sur les chaînes publiques de Radio-Canada et de RadioQuébec depuis 1985.

6 Cette recherche a été réalisée grâce à une subvention du vice-rectorat aux ressources humaines de l'Université Laval et avec l'aide indispensable du FCAR.

\section{OUVRAGES CITÉS}

Becker, Howard Saul. Les Mondes de l'art. Paris: Flammarion, 1988.

Borie, Bertrand. "Entretien avec Giorgio Moroder". L'Ecran fantastique, n" 47 (1984), p. 68-69.

Boudon, Raymond. "De l'objectivité des valeurs artistiques ou les valeurs artistiques entre le platonisme et le conventionnalisme". Archives de philosophie du droit, vol. 40 (1995), p. 76-95.

Brion, Patrick. "Lang (Fritz)». Encyclopaedia Universalis, tome 13. Paris: Encyclopédie Universalis (1989), p. 432-434.

Buñuel, Luis. Mon dernier soupir. Paris: Ramsay, 1986.

Cieutat, Brigitte. "Fritz Lang "morodernisé" ou l'art du détournement ». Positif, n" 285 (1984), p. 12-14.

Collectif. Fantastique et réalisme dans le cinéma allemand 1912-1933. Bruxelles: Musée du cinéma, 1969.

Courtade, Francis. Cinéma expressionniste. Paris: Veyrier, 1984.

Eibel, Alfred. Fritz Lang. Trois Lumières. Paris: Flammarion, 1988.

Eisner, Lotte H. Fritz Lang. Paris: Flammarion, 1988.

Goffman, Erving. Les Cadres de l'expérience. Paris: Minuit, 1991.

Guback, Thomas. "Derrière les ombres de l'écran: le cinéma hollywoodicn en tant qu'industrie». Sociologie et sociétés, vol. 8, n" 1 (1976), p. 5-24. 
Guibert, Hervé. "Firitz Lang défiguré ou magnifié?". Le Monde, 8 août 1984, p. 11.

Kracauer, Siegfried. De Caligari à Hitler. Une histoire psychologique du cinéma allemand. Paris: Flammarion, 1988.

Laberge, Yves. "Une mémoire filmique défaillante: oublis et plans manquants dans la version sonorisće (1984) du film Métropolis (1927) de Fritz Lang". Champs visuels. Revue interdisciplinaire de recherches sur l'image, n" 4 (1997), p. 135-146.

Labcrge, Yves. "Nouvelle technologie, nouvelle idéologie", dans Martine Époque (dircction), Arts et technologies. Lyon: Éditions du Centre (1995), p. 125-129.

Laberge, Yves. "Trois études sur un film de Fritz Lang". Communication information, vol. 15, n"1 (1994), p. I13-122.

Laberge, Yves. "Le retour de Métropolis: une idéologie subversive au service d'une autre". Etudes littéraires, vol. 18, n" 1 (1985), p. 133-156.

Marie, Michel. M le matudit. Etude critique. Paris: Nathan, 1990.

Metz, Christian. L'Énonciation impersonnelle ou le site du film. Paris: MéridiensKlincksieck, 1991.

Moulin, Raymonde. "Art (sociologie de l')", dans Raymond Boudon (direction), Dictionnaire de la sociologie. Paris: Larousse (1993), p. 20-21.

Odin, Roger. "Du spectateur fictionnalisant au nouveau spectateur: approche sémiopragmatique". Iris, n" 8 (1988), p. 121-139.

Passek, Jean-Loup (direction). Dictionnaire du cinéma. Paris: Larousse, 1991.

Passek, Jean-Loup (direction). 20 ans de cinéma allemand. Paris: Centre GeorgesPompidou, 1978.

Tulard, Jean (direction). Givide des films, tome 2. Paris: Laffont, 1990. 